Check for updates

Cite this: RSC Adv., 2017, 7, 32727

\title{
Microbeads preparation with reversed-phase microemulsion and ultraviolet light curing for digital encoding suspension array $\dagger$
}

\author{
Q. H. He, ${ }^{a b}$ D. M. Li, ${ }^{a b}$ Y. H. He, (D)*ab Y. L. Zhang, ${ }^{a b}$ Z. Y. Shen, ${ }^{a b}$ L. Zhu, $^{\text {ab }}$ X. J. Chen, ${ }^{a b}$ \\ S. Y. Liu ${ }^{a}$ and F. Y. Chen ${ }^{\star c}$
}

\begin{abstract}
We provided a microbeads preparation method with reversed-phase microemulsion and ultraviolet light curing for laser induced breakdown spectroscopy based digital encoding suspension array. By combining reversed-phase microemulsion and ultraviolet light curing together, we created an one-step system for preparation of microbeads. The stability and universality of this method had been confirmed in preparation of microbeads with different encoding materials. With further experiments in surface modification and multiplexed detection, we proved that the synthesized microbeads were available to prepare suspension array and could provide accurate encoding performance in multiplexing.
\end{abstract}

Received 21st January 2017

Accepted 21st June 2017

DOI: $10.1039 / \mathrm{c} 7 \mathrm{ra00894e}$

rsc.li/rsc-advances

also used as labels of target biomolecules, the cross talk can also

\section{Introduction}

In life science research, suspension array has become an increasingly attractive detection method because of its performance in multiplexing and high throughput detection..$^{1-4}$ For example, suspension array achieves lower detection limits and higher sensitivity in clinical diagnosis than traditional technology. ${ }^{5,6}$ In genome and protein analysis, suspension array also proves its high capability in multiplexing. ${ }^{7}$ Most of above characters of suspension array in detection rely on the encoding performance of microbeads, the carriers of suspension array. The current encoding system of suspension array is based on multi-color fluorescence barcodes. ${ }^{8,9}$ Operationally, researchers assemble organic fluorophores or luminescent quantum-dots with functional microbeads to prepare suspension array. ${ }^{10}$ However, limited by the light-emitting principle and characteristics, the fluorescence based encoding method is faced with several disadvantages in detection. First of all, the utilization of multi-color fluorescent materials always causes spectral overlaps due to the broad waveform, which is a challenge to the accuracy of coding. ${ }^{11}$ Then, because of the existence of fluorescence resonance energy transfer, the cross talk always happen between different color, hurting the stability of coding..$^{12}$ Most important of all, since fluorescent materials are

\footnotetext{
${ }^{a}$ Shenzhen Key Laboratory for Minimal Invasive Medical Technologies, Institute of Optical Imaging and Sensing, Graduate School at Shenzhen, Tsinghua University, Shenzhen 518055, China

${ }^{b}$ Department of Physics, Tsinghua University, Beijing 100084, China

'Department of Biomedical Engineering, Southern University of Science \& Technology, Shenzhen 518055, China. E-mail: chenfy@sustc.edu.cn

$\dagger$ Electronic supplementary information (ESI) available. See DOI: 10.1039/c7ra00894e
} happen between fluorescence based encoding signal and label signal, which not only do harm to the characteristic of encoding, but also produce indeterminacy in the whole detection procedure. $^{13}$

In our previous research, we provided the digital encoding method for suspension array with laser induced breakdown spectroscopy (LIBS). ${ }^{14}$ LIBS is widely utilized in industry analysis, environment monitoring and medicine analysis as an elemental measurement method. ${ }^{15,16}$ After stimulation by laser pulse, plasma can be produced on the surface of samples. We can obtain the laser induced breakdown spectra by collecting the emissions of plasma. In the laser induced breakdown spectra, the existence of specific peaks can demonstrate the existence of corresponding elements in the sample. ${ }^{17}$ With this technology, we assembled the encoding materials which include specific elements on the carrier to prepare encoded suspension array. In the decoding procedure, the suspension array experienced the stimulation of pulse laser and emitted laser induced breakdown spectra with stable, sharp and readable emission peaks. Using character 1 to represent the existence of specific peak and 0 to represent the opposite, we created a series of binary sequences to represent the corresponding microbeads, achieving the digital encoding of suspension array.

In this paper, we provide a microbeads preparation method with reversed-phase microemulsion and ultraviolet light curing for suspension array. Reversed-phase microemulsion method is widely adopted in the synthesis of particles with different sizes. ${ }^{18}$ By configurating a stable microemulsion with aqueous solvent, oil solvent and enough surfactant, the disperse phase can be incised by continuous phase and maintained as liquid microbeads. Once the disperse phase can be solidified, microbeads can be formed and extracted from the dispersion. Ultraviolet light curing is 


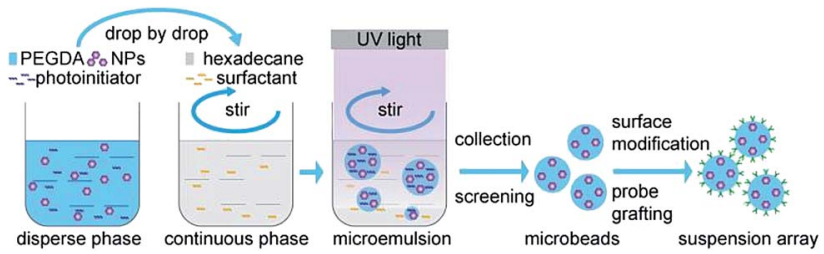

Fig. 1 The synthesis scheme of digital encoding suspension array using reversed-phase microemulsion and ultraviolet light curing.

a kind of photochemical reaction which is usually utilized in film process and printing technology. Briefly, after the exposure under ultraviolet light, the low molecular materials can form polymer by polymerization and cross linking, which always appears as solidification of liquid materials..$^{19}$ In our experiments, we used metal and metallic oxide nanoparticles to provide encoding signals. We mixed encoding materials with polyethylene glycol acrylate (PEGDA), the aqueous solvent, and 2-hydroxy-4'-(2hydroxyethoxy)-2-methylpropiophenone, the photoinitiator, to play the role of disperse phase. The disperse phase was dropwise added into continuous phase which was prepared by hexadecane, the oil solvent, and EM-90, the surfactant. The dispersion was continuously stirred and exposed under ultraviolet light to achieve the solidification. After collection and screening, microbeads with uniform sizes were picked out to experience the surface modification and probe grafting to prepare suspension array. The scheme of the whole process was shown in Fig. 1.

\section{Experimental section}

\section{Reagent}

PEGDA (average Mn $=250$ ), EM-90, hexadecane were purchased from Sigma-Aldrich. Silver $(\mathrm{Ag})$, cuprous oxide $\left(\mathrm{Cu}_{2} \mathrm{O}\right)$, magnesium oxide $(\mathrm{MgO})$, zinc oxide $(\mathrm{ZnO})$ nanoparticles, dopamine hydrochloride and 2-hydroxy-4'-methylpropiophenone-(2hydroxyethoxy)-2-methylpropiophenone were purchased from Macklin reagent, China. Bull serum albumin (BSA) and IgG was purchased from Beyotime Biotechnology, China. Anti-IgG (labeled with QDs) were purchased from Wuhan Jiayuan Quantum Dot Technological Development Corporation, China.

\section{Preparation of encoded microbeads}

The disperse phase was composed of PEGDA, nanoparticles and 2-hydroxy-4'-(2-hydroxyethoxy)-2-methylpropiophenone. $20 \mathrm{mg}$ photoinitiator were dispersed in $1 \mathrm{~mL}$ PEGDA and experience vigorous stirring and ultrasonication to form stable dispersion. $1 \mathrm{~mL}$ nanoparticles solution $\left(2 \mathrm{mg} \mathrm{mL}^{-1}\right.$, water) was centrifuged and the supernatant was removed. The nanoparticles were redispersed in the prepared dispersion by ultrasonication to form disperse phase solution. The continuous phase was prepared by mixing EM-90 with hexadecane in a ratio of $1: 20$. The disperse phase was poured into continuous phase dropwise and dispersed by magnetic stirring. The stirring microemulsion was exposed under the ultraviolet light for 6 hours to achieve the solidification. The intensity of UV light in curing was measured by optical power meter to be $7 \mathrm{~mW} \mathrm{~cm}^{-2}$. Solidified microbeads were washed with deionized water and pure ethanol for three times. After collection and screening, the microbeads with similar sizes were picked out to be ready for further modification.

\section{Surface modification of encoded microbeads}

Dopamine was utilized as functional group provider to achieve the surface modification of microbeads. Specifically, dopamine was dissolved in Tris- $\mathrm{HCl}$ buffer solution $(10 \mathrm{mM}, \mathrm{pH}=8.5)$ in the concentration of $2 \mathrm{mg} \mathrm{mL}^{-1}$. The microbeads were added into the dopamine solution and the dispersion was stirred for $24 \mathrm{~h}$ at room temperature. The modified microbeads were washed with deionized water for several times before probe grafting.

\section{Probe grafting and multiplexed detection}

We carried out probe grafting on the modified surface of microbeads to prepare suspension array. Then, a series of antigen-antibody binding experiments were carried to illustrate the availability of suspension array in practical multiplexed detection. With $\mathrm{Ag}$ and $\mathrm{Cu}_{2} \mathrm{O}$ nanoparticles, we prepared two types of microbeads: MB1 (AgNPs) and MB2 $\left(\mathrm{Cu}_{2} \mathrm{ONPs}\right)$. Corresponding to two types of microbeads, we chose two antigenantibody combinations to accomplish the specific detection process, list as follow: (1) mouse IgG and $525 \mathrm{~nm}$ QDs labeled goat anti-mouse IgG; (2) rabbit IgG and $605 \mathrm{~nm}$ QDs labeled goat anti-rabbit IgG. After surface modification with dopamine solution, microbeads were redispersed in ultrapure water in the concentration of $10 \mathrm{mg} \mathrm{mL}^{-1} 100 \mu \mathrm{L}$ microbeads solution was added into $1 \mathrm{~mL}$ phosphate buffer solution (PBS, $50 \mathrm{mM}, \mathrm{pH}$ 7.4) and stirred for 15 minutes at room temperature. Then, 10 $\mu \mathrm{L}$ IgG solution $\left(1 \mathrm{mg} \mathrm{mL}^{-1}\right)$ and $50 \mu \mathrm{L} \mathrm{EDC}\left(10 \mathrm{mg} \mathrm{mL}^{-1}\right)$ was added in the dispersion and incubated for 2 hours at $37^{\circ} \mathrm{C}$. In this step, MB1, 2 were reacted with mouse IgG and rabbit IgG correspondingly to form different suspension array. Suspension array were washed with PBS for several times and redispersed in $1 \mathrm{~mL}$ PBS, 5\% BSA was used as blocking solution to cover excess amino. Reacted suspension array were washed and redispersed in $1 \mathrm{~mL}$ PBS, and $10 \mu \mathrm{L}$ anti-IgG solution $(50 \mathrm{mM})$ was added and stirred for 30 minutes at $37^{\circ} \mathrm{C}$, the final suspension array were washed with PBS to remove excess labeled anti-IgG and redispersed in PBS.

\section{Results and discussions}

\section{Principle of LIBS based digital encoding}

Based on above statement, we used four types of nanoparticles, including AgNPs, $\mathrm{Cu}_{2} \mathrm{ONPs}$, MgONPs and ZnONPs, to work as encoding materials. The initial LIBS spectra of these encoding materials were stimulated and collected by our home made LIBS optical system (Fig. 1S $\dagger$ ), and they were shown in Fig. 2. In our measurement wavelength range, we picked out 8 most strong signal peaks to be the encoding signal. Since the specific peaks can represent the existence of corresponding materials, we can use 1 and 0 to represent the existence of encoding materials in suspension array. In other words, the suspension 


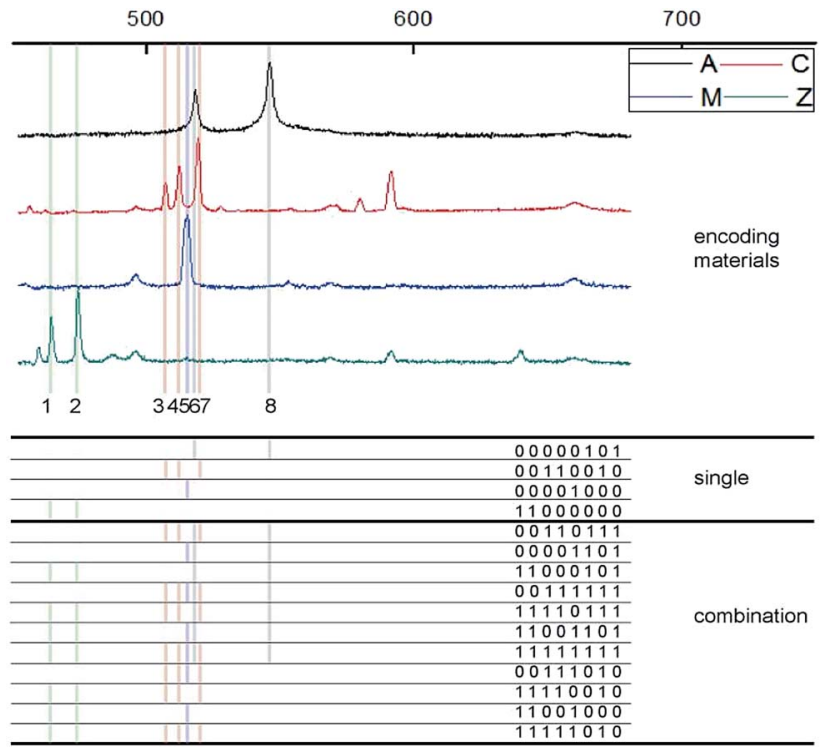

Fig. 2 The LIBS spectra of initial nanoparticles and principle of digital encoding.

array can be labeled with specific digital binary sequence, accomplishing the digital encoding. Furthermore, through the composite utilization of different types of encoding materials, we can create a much bigger number of encoding amount than the initial number of materials types. The encoding amount can be explained with this formula:

$$
W=\mathrm{C}_{n}{ }^{1}+\mathrm{C}_{n}{ }^{2}+\ldots+\mathrm{C}_{n}{ }^{n}=2^{n}-1
$$

$W$ represents the amount of encoding; $n$ represents the number of material types. It is predictable that the amount of encoding can get an exponential growth with the addition of new encoding materials.

\section{Characterization of synthesized microbeads}

We carried out a series of measurements to describle the synthesis of microbeads. The solidified microbeads after screening were shown in Fig. 3a and b. In the inset of Fig. 3b, the encoded microbeads showed different appearance with unencoded microbeads, which indicated the nanoparticle had been actually enveloped in microbeads. We prepared 4 kinds of microbeads with our encoding materials and collected their LIBS spectra with our LIBS system, the results are shown in Fig. 3c. A, C, M, Z represent AgNPs, $\mathrm{Cu}_{2} \mathrm{ONPs}, \mathrm{MgONPs}$ and ZnONPs respectively, different combinations of letters represent microbeads encoded by different combinations of nanoparticles. Despite of the emission peaks caused by the basement, the encoding peaks matched well with initial nanoparticles, indicating that the encoding materials were well coupled in microbeads. We used particle size measurement software (nano Measurer 1.2) to analysis the size distribution of microbeads. As shown in Fig. 3e, the measurement of size distribution numerically demonstrated that the synthesized microbeads are in similar sizes. Corresponding to different concentrations of photoinitiator in preparations of 4 kinds of

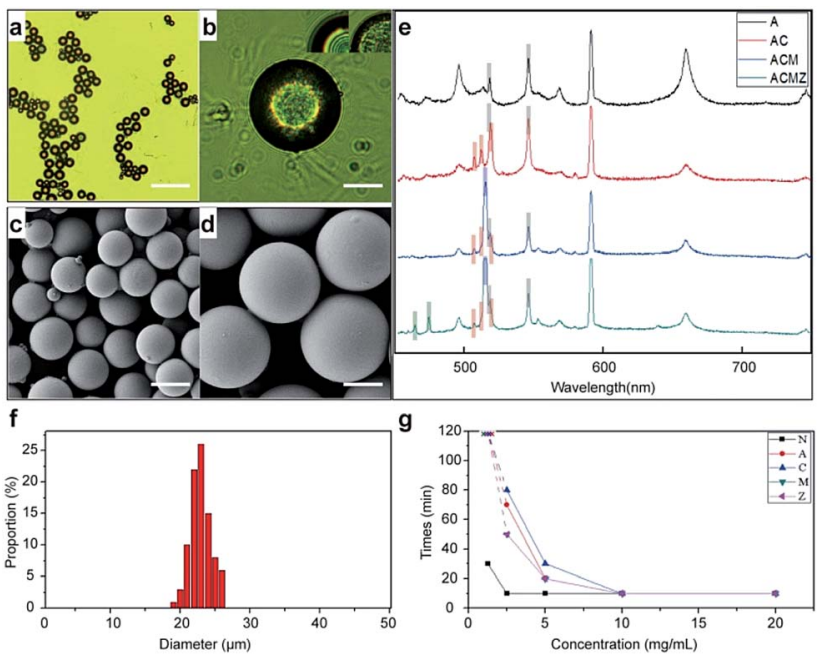

Fig. 3 The characteristic of synthesized microbeads. (a, b) Microscope images of microbeads. The insets in (b) are the comparison between unencoded microbeads and encoded microbeads (c, d) scanning electron microscope images of microbeads. Scale bars are $100 \mu \mathrm{m}$ in (a), $10 \mu \mathrm{m}$ in (b), $25 \mu \mathrm{m}$ in (c), $10 \mu \mathrm{m}$ in (d). (e) The LIBS spectra of 4 kinds of microbeads. (f) The size distribution of microbeads. (g) The curing time of 4 kinds of microbeads with different concentrations of photoinitiator.

microbeads and no-coding microbeads, the curing time is measured and recorded in Fig. 3g. It is verified that the dosage of photoinitiator is a key element to curing efficiency.

\section{Characterization of surface modified microbeads}

As the carrier of suspension array, microbeads need to be modified on the surface to cover enough functional group to offer binding sites for bioprobes. Dopamine is usually used in surface modification because of its strong stickiness and abundant amino and carboxyl. ${ }^{20}$ We treated our prepared microbeads with the Tris- $\mathrm{HCl}$ solution of dopamine, the comparison of initial microbeads and dopamine coated microbeads was shown in Fig. 4. The rough surface of microbeads indicates that the dopamine had been polymerized on the microbeads.

\section{Application of suspension array in multiplexing}

To demonstrate the appearance of synthesized suspension array in practical multiplexed bimolecular detection, we carried out antibody detection experiment with two types of encoded suspension array. Mouse IgG and rabbit IgG were grafted on MB1 and MB2 as the bioprobe of green QDs labeled goat anti mouse IgG and red QDs labeled goat anti rabbit IgG respectively. Two types of suspension array were mixed and accomplished target detection together. The fluorescent microscope image of reacted suspension array was shown in Fig. 5a. It is clear that microbeads were covered with two different colors and almost each microbead was covered with one color, which indicates that specific detection had been achieved by our suspension array. As shown in Fig. 5b, the encoding signal and label fluorescence of two types of reacted suspension array were 


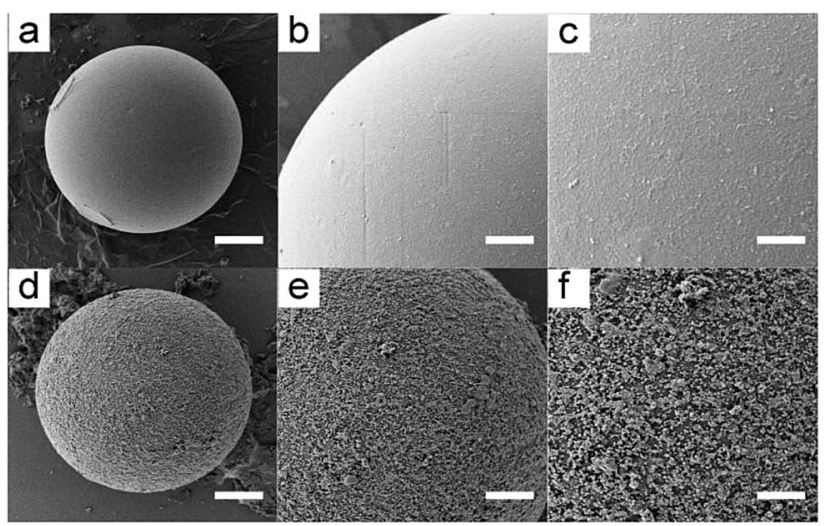

Fig. 4 The comparison between initial microbeads and dopamine modified microbeads. $(a, b, c)$ Scanning electron microscope images of initial microbeads. (d, e, f) Scanning electron microscope images of dopamine modified microbeads. Scale bars are $6 \mu \mathrm{m}$ in (a, d), $3 \mu \mathrm{m}$ in (b, e), $1.5 \mu \mathrm{m}$ in (c, f).

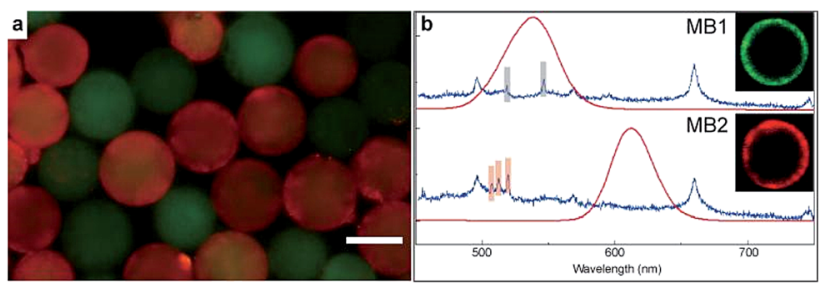

Fig. 5 The multiplexing of suspension array (MB1, MB2). (a) The fluorescent microscope image of reacted suspension array, the scale bar is $20 \mu \mathrm{m}$. (b) The encoding signal and label fluorescence spectrum of reacted MB1 and MB2, the insects are cross section images of laser confocal microscope of reacted MB1 and MB2.

collected independently to show the corresponding relationship between encoding and analytes types. The insects of Fig. 5b were cross section images of laser confocal microscope of reacted suspension array. The green and red rings are the label of analytes, besides this, the other parts of suspension array are dark, which means that the fluorescence background was eliminated so that the cross talk between label and encoding signal can be avoided with our prepared suspension array.

\section{Conclusions}

In conclusion, we had provided a stable preparation method for laser induced breakdown spectroscopy based digital encoding suspension array. By combining reversed-phase microemulsion and ultraviolet light curing together, we created a one-step system for preparation of microbeads. The stability and universality of this method had been confirmed in preparation of microbeads with different encoding materials. With further experiments in surface modification and multiplexed detection, we proved the availability of suspension array in practical application. Based on the simplified preparation process, wide suitability and stable encoding performance, it is reasonable to declare that the reversed-phase microemulsion and ultraviolet light curing method is available and creative in preparation of digital encoding suspension array.

\section{Notes and references}

1 Y. Lu, J. Zhao, R. Zhang, Y. Liu, D. Liu, E. M. Goldys, X. Yang, P. Xi, A. Sunna, J. Lu, Y. Shi, R. C. Leif, Y. Huo, J. Shen, J. A. Piper, J. P. Robinson and D. Jin, Nat. Photonics, 2014, 8, 32-36.

2 F. Wang, R. Deng, J. Wang, Q. Wang, Y. Han, H. Zhu, X. Chen and X. Liu, Nat. Mater., 2011, 10, 968-973.

3 S. Brenner, M. Johnson, J. Bridgham, G. Golda, D. H. Lloyd, D. Johnson, S. Luo, S. McCurdy, M. Foy, M. Ewan, R. Roth, D. George, S. Eletr, G. Albrecht, E. Vermaas, S. R. Williams, K. Moon, T. Burcham, M. Pallas, R. B. DuBridge, J. Kirchner, K. Fearon, J.-i. Mao and K. Corcoran, Nat. Biotechnol., 2000, 18, 630-634.

4 C. Lin, R. Jungmann, A. M. Leifer, C. Li, D. Levner, G. M. Church, W. M. Shih and P. Yin, Nat. Chem., 2012, 4, 832-839.

5 R. Bellisario, R. J. Colinas and K. A. Pass, Clin. Chem., 2000, 46, 1422.

6 S. J. Wong, V. L. Demarest, R. H. Boyle, T. Wang, M. Ledizet, K. Kar, L. D. Kramer, E. Fikrig and R. A. Koski, J. Clin. Microbiol., 2004, 42, 65-72.

7 J. P. Nolan and L. A. Sklar, Trends Biotechnol., 2002, 20, 9-12. 8 S. V. Vaidya, M. L. Gilchrist, C. Maldarelli and A. Couzis, Anal. Chem., 2007, 79, 8520-8530.

9 X. Michalet, F. F. Pinaud, L. A. Bentolila, J. M. Tsay, S. Doose, J. J. Li, G. Sundaresan, A. M. Wu, S. S. Gambhir and S. Weiss, Science, 2005, 307, 538.

10 S. P. Perfetto, P. K. Chattopadhyay and M. Roederer, Nat. Rev. Immunol., 2004, 4, 648-655.

11 M. Han, X. Gao, J. Z. Su and S. Nie, Nat. Biotechnol., 2001, 19, 631-635.

12 Biophotonics, Part B. Methods in Enzymology, ed. G. Marriott and I. Parker, Academic Press, San Diego, CA, 2003, vol. 361.

13 Y. Liu, L. Liu, Y. He, L. Zhu and H. Ma, Anal. Chem., 2015, 87, 5286-5293.

14 Q. He, Y. Liu, Y. He, L. Zhu, Y. Zhang and Z. Shen, Sci. Rep., 2016, 6, 36511.

15 B. Kearton and Y. Mattley, Nat. Photonics, 2008, 2, 537-540.

16 V. Sautter, M. J. Toplis, R. C. Wiens, A. Cousin, C. Fabre, O. Gasnault, S. Maurice, O. Forni, J. Lasue, A. Ollila, J. C. Bridges, N. Mangold, S. Le Mouelic, M. Fisk, P. Y. Meslin, P. Beck, P. Pinet, L. Le Deit, W. Rapin, E. M. Stolper, H. Newsom, D. Dyar, N. Lanza, D. Vaniman, S. Clegg and J. J. Wray, Nat. Geosci., 2015, 8, 605-609.

17 D. W. Hahn and N. Omenetto, Appl. Spectrosc., 2012, 66, 347419.

18 X. Wang, Y. Jiang, Y.-W. Wang, M.-T. Huang, C.-T. Ho and Q. Huang, Food Chem., 2008, 108, 419-424.

19 F. Bauer, R. Flyunt, K. Czihal, H. Langguth, R. Mehnert, R. Schubert and M. R. Buchmeiser, Prog. Org. Coat., 2007, 60, 121-126.

20 H. Lee, S. M. Dellatore, W. M. Miller and P. B. Messersmith, Science, 2007, 318, 426. 